\title{
In-Depth Genomic Characterization of a Meropenem-nonsusceptible Pseudomonas otitidis Strain Contaminating Chicken Carcass
}

\author{
Tatiana Regina Vieira', Gustavo Enck Sambrano', Núbia Michelle Vieira da Silva², \\ Priscylla Carvalho Vasconcelos ${ }^{2}$, Esther Ferraza Cavinatto de Oliveira' ${ }^{1}$, Celso José Bruno de Oliveiraa ${ }^{2,4}$, \\ Samuel Paulo Cibulski² \& Marisa Cardoso'
}

\begin{abstract}
Background: The indiscriminate use of antibiotics in food-animal production has a major impact on public health, particularly in terms of contributing to the emergence and dissemination of antimicrobial resistant bacteria in the food-animal production chain. Although Pseudomonas species are recognized as important spoilage organisms in foodstuff, they are also known as opportunistic pathogens associated with hospital-acquired infections. Furthermore, Pseudomonas can play a role as potential reservoirs of antimicrobial resistance genes, which may be horizontally transferred to other bacteria. Considering that cephalosporins ( $3 \mathrm{rd}$ and higher generations) and carbapenems are critically important beta-lactam antimicrobials in human medicine, this study reports the occurrence and genomic characterization of a meropenem-nonsusceptible Pseudomonas otitidis strain recovered from a chicken carcass in Brazil.

Materials, Methods \& Results: During the years 2018-2019, 72 frozen chicken carcasses were purchased on the retail market from different regions in Brazil. Aliquots from individual carcass rinses were screened for Extended Spectrum Beta-lactamase (ESBL)-producing bacteria in MacConkey agar supplemented with $1 \mathrm{mg} . \mathrm{L}^{-1}$ cefotaxime. Phenotypically resistant isolates were further tested for resistance to other antimicrobials and confirmed as ESBL-producers by means of disk-diffusion method using Müller-Hinton agar. Only one meropenen-nonsusceptible isolate was detected and submitted to whole genome sequencing (WGS) in Illumina Miseq. The strain was identified as Pseudomonas otitidis by local alignment of the 16S rRNA sequence using BLASTn and confirmed by Average Nucleotide Identity (ANI) analysis using JspeciesWS database. Genes encoding for antimicrobial resistance were detected by means of Resfinder and Comprehensive Antibiotic Resistance Database (CARD) databases. The phenotypic non-susceptibility to meropenen was attributed to the gene $b l a_{\text {РОM-1 }}$. A total of 192 different genes encoding for quorum sensing system, antiphagocytosis, iron uptake, efflux pump, endotoxin and toxin, adherence, and secretion systems were detected by means of Virulence Factor Database (VFDB). Pseudomonas otitidis-pan genome was built using Roary-rapid large-scale prokaryote pan genome analysis using the present strain (K_25) and other two P. otitidis genomes (PAM-1, DSM 17224) publicly available at the NCBI. The core genome analysis of the two human strains resulted in similar percentages.

Discussion: Carbapenems are critically important drugs for human health and bacterial strains resistant to these antimicrobials pose a public health problem. The bla $a_{\mathrm{POM}-1}$ gene harbored by the Pseudomonas otitidis $\mathrm{K} \_25$ strain encodes a metallo-beta-lactamase (MBL) conferring resistance to carbapenems. Pseudomonas otitidis was the first confirmed pathogenic Pseudomonas species expressing MBL constitutively in the absence of inducible beta-lactamase genes. Furthermore, the several virulence genes associated with the capacity of the P. otitidis $\mathrm{K} \_25$ to colonize, evade the immune system and cause lesions in the human host confirm this strain as a potential opportunistic pathogen contaminating foodstuff. These reinforce the need to address antimicrobial resistance in a One Health perspective, in which resistant bacteria and resistance determinants circulate among environment, animals and humans.
\end{abstract}

Keywords: carbapenem-resistance, POM-1, Pseudomonas otitidis, Brazil.

Descritores: resistência bacteriana, Meropenem, Pseudomonas otitidis, Brasil.

DOI: $10.22456 / 1679-9216.103176$

Received: 3 March 2020

Accepted: 4 June 2020

Published: 3 July 2020

${ }^{1}$ Departamento de Medicina Veterinária Preventiva, Faculdade de Medicina Veterinária, Universidade Federal do Rio Grande do Sul (UFRGS), Porto Alegre, RS, Brazil. ${ }^{2}$ Laboratório de Avaliação de Produtos de Origem Animal (LAPOA), Departamento de Zootecnia, Centro de Ciências Agrárias \& ${ }^{3}$ Laboratório de Biotecnologia Celular e Molecular, Centro de Biotecnologia (Cbiotec), Universidade Federal da Paraíba (UFPB), Areia, PB, Brazil. ${ }^{4}$ Global One Health Initiative (GOHi), The Ohio State University, Columbus, OH, USA. CORRESPONDENCE: T.R. Vieira [tatianareginavieira@gmail.com]. Faculdade de Medicina Veterinária - UFRGS. Av. Bento Gonçalves n. 9090. CEP 91540-000 Porto Alegre, RS, Brazil. 


\section{INTRODUCTION}

Pseudomonas are opportunistic pathogens often related to complicated nosocomial infections in humans. Moreover, Pseudomonas species harbor intrinsic and acquired antimicrobial resistance mechanisms, leading to hard-to-treat infections [16] with severe disease outcome as a result of various virulence factors such as flagellum, pili, protein secretion systems, exoenzymes, lectins, quorum sensing and biofilm matrix synthesis [9,10,24].

In foods, Pseudomonas species are recognized as important spoilage bacteria [22] causing significant economic losses in the food industry [18]. Among these species, Pseudomonas otitidis was first described in patients diagnosed with acute otitis [6], and has thereafter been isolated from environmental sites and foodstuffs [15,23,28,29].

The majority of the studies about antimicrobial resistance in foods address only foodborne pathogens and microbial indicators, such as Escherichia coli and Enterococcus sp. Therefore, the role of spoilage bacteria as potential reservoirs of antimicrobial resistance genes that can be transmitted to other bacteria is practically untapped. Among the antimicrobials of the beta-lactam class, cephalosporins (3rd and higher generation) and carbapenems are critically important drugs in human medicine, as bacterial pathogens that are resistant to these antimicrobial agents pose a public health concern globally [26]. Therefore, information regarding the mechanisms associated with bacterial resistance against these drugs is critical to tackle the emergence and dissemination of antimicrobial resistance. This study aimed at providing in-depth genomic characterization of a meropenem-nonsusceptible Pseudomonas otitidis recovered from chicken carcass in Brazil.

\section{MATERIALS AND METHODS}

\section{Samples}

During the years 2018-2019, 72 frozen chicken carcasses were purchased on retail market from different cities in Brazil in order to investigate the occurrence of Extended Spectrum Beta-lactamases (ESBL) producing- and carbapenem-resistant enterobacteria. Frozen chicken carcasses were collected in their original packaging and kept refrigerated at $4^{\circ} \mathrm{C}$ for defrosting before processing. After the removal of the plastic package and the giblets, the carcasses were placed into sterile plastic bags and weighted. Four hundred milliliters of buffered peptone water (BPW 1\%) were added into the bag, and the carcass was rinsed for approximately one minute.

\section{Screening for ESBL and resistance to carbapenems}

Aliquots $(30 \mathrm{~mL})$ from the carcass-rinses were cultured in non-selective enrichment broth (BPW $1 \%$ ) at $37^{\circ}$ for $18-22 \mathrm{~h}$. Thereafter, one loopful $(10 \mu \mathrm{L})$ of the overnight culture was transferred to MacConkey ${ }^{1}$ agar plates containing $1 \mathrm{mg} . \mathrm{L}^{-1}$ of cefotaxime $^{2}$ (CTX agar) and incubated at $44^{\circ} \mathrm{C} \pm$ $0.5^{\circ} \mathrm{C}$ for $18-22 \mathrm{~h}$. Up to three colonies per sampled carcass were individually picked, streaked onto CTX agar and cultivated at $37^{\circ} \mathrm{C} \pm 1{ }^{\circ} \mathrm{C}$ for $18-22 \mathrm{~h}$. In order to screen for ESBL producing- and carbapenem resistant bacteria, each isolate was tested for antimicrobial susceptibility to cefotaxime $(30 \mu \mathrm{g})$, ceftazidime $(30 \mu \mathrm{g})$ and meropenem $(10 \mu \mathrm{g})$ disks ${ }^{1}$ by means of disk-diffusion method using MüllerHinton $^{1}$ agar [7,8]. Escherichia coli ATCC $^{\odot} 25922$ was used for quality control purposes.

\section{Isolates identification and antimicrobial susceptibility test}

The isolates were phenotypically identified by means of biochemical procedures as previously described [17]. Further antimicrobial resistance profiling was determined by disk-diffusion test, against the following drugs ${ }^{1}$ : amikacin $(30 \mu \mathrm{g})$, ciprofloxacin $(5 \mu \mathrm{g})$, imipenem $(10 \mu \mathrm{g})$, gentamicin $(10 \mu \mathrm{g})$, levofloxacin $(5 \mu \mathrm{g})$, norfloxacin $(10 \mu \mathrm{g})$ and tobramycin $(10 \mu \mathrm{g})$. The minimum inhibitory concentration (MIC) of colistin ${ }^{2}$ was determined by broth microdilution method using cation-adjusted Muller-Hinton broth ${ }^{1}$ (CAMHB) [7].

\section{Sequencing and analysis}

Genomic DNA was extracted using MagMAXTM CORE Nucleic Acid Purification $\mathrm{Kit}^{3}$ according to the manufacturer's instructions. DNA integrity was visually assessed on $1 \%$ agarose gel and quantified by fluorometry (Qubit $)^{4}$. The genomic library was prepared by means of Nextera XT DNA Library ${ }^{5}$. Fragment sizes were evaluated using a capillary electrophoresis system (Fragment Analyzer) ${ }^{6}$, and paired-end sequenced in Illumina MiSeq ${ }^{5}$ using a 2x250 cycle V2 kit $^{5}$. 
Fastqc tool (bioinformatics.babraham.ac.uk/ projects/fastqc) was used to check the quality of the reads, followed by trimming of Illumina adapters and low-quality reads (Phred score $<30$ ) using Trimmomatic (available from usadellab.org/cms/index). Genome assembly was performed using SPAdes [1] and Abyss [21]. The assembly quality was assessed in QUAST [11] and the best result was obtained using SPAdes with a k-mer of 77 . In order to improve the assembly metrics in terms of N50, we used the Aligngraph tool [2]. Before running AlignGraph tool, a dotplot similarity matrix by means of pairwise alignments was obtained using the web platform DGENIES [5].

The strain was identified by local alignment of the 16S rRNA sequence using BLASTn and confirmed by Average Nucleotide Identity (ANI) analysis using JspeciesWS database [19] (available from jspecies. ribohost.com). Barrnap (http://www.vicbioinformatics. com/software.barrnap.shtml) was used to extract the 16S rRNA sequence for identification. The genome annotation was performed under the automatic NCBI pipeline submission system. Roary-rapid large-scale prokaryote pan genome analysis (academic.oup.com/ bioinformatics/article-lookup/doi/10.1093/bioinformatics/btv421) was used to build a pangenome.

Genes encoding for antimicrobial resistance were screened by means of ResFinder 3.2 (available from cge.cbs.dtu.dk/services/ResFinder/) and CARD (available from https://card.mcmaster.ca/analyze/rgi). Virulence factors were investigated using the Virulence Factor Database (VFDB) [13].

\section{RESULTS}

From the 72 frozen chicken carcasses, 55 (76.4\%) were positive for microbiological isolation in CTX agar. A total of 164 colonies were screened for resistance to meropenem and cephalosporins. After screening, only one isolate showed non-susceptibility to meropenem, while it was susceptible to all tested cephalosporins. This isolate was phenotypically assigned within the Pseudomonas genus. Further determination of antimicrobial resistance profiling demonstrated that the isolate was susceptible to amikacin, ciprofloxacin, imipenem, gentamicin, levofloxacin, norfloxacin and tobramycin. The MIC of colistin was determined at $2 \mathrm{mg} . \mathrm{L}^{-1}$, below the resistance breakpoint $\left(\geq 4 \mathrm{mg} \cdot \mathrm{L}^{-1}\right)$ [8].
Since meropenem-resistance is not a frequent feature among bacteria from foodstuffs, we decided to further characterize the strain, which was registered in the culture collection as $\mathrm{K} \_25$. The whole genome sequencing (WGS) was performed and the genome sequence was estimated as $6,406,500$ bp long with $67.06 \%$ of GC (Table 1).

The local alignment of the 16S rRNA sequence using BLASTn identified the strain K_25 as Pseudomonas otitidis. The Average Nucleotide Identity (ANI) resulted in a 0.99 cutoff value compared with the reference $P$. otitidis, indicating that the strain was correctly identified. The genome annotation was performed under the automatic NCBI pipeline submission system. The draft genome sequence of $P$. otitidis $\mathrm{K} \_25$ is available at GenBank under the accession no. NZ_WTFN00000000.

There were only two available Pseudomonas otitidis strains (PAM1 and DSM17224) available at the NCBI database: DSM 17224 - FOJP00000000 isolated in 2016 in the United States; and PAM-1PXJI00000000 isolated in 2018 in Lebanon, both from humans. The results demonstrated that the $P$. otitidis K_25 genome was not very close to any of the two references. As expected, AlignGraph results did not produce any extension of the contigs.

The pangenome was built with the present strain (K_25) and the other two reference strains (PAM1, DSM 17224). The obtained pangenome consisted of a total of 7,463 genes while the core genome presented a total of 4,595 genes, which comprised $71.25 \%$ of the entire genome of the K_25 strain. The analysis of the two human strains resulted in similar percentages. In addition, P. otitidis K_25 was found to harbor a 2,389 bp plasmid. The cryptic plasmid had a GC content of $60.7 \%$ and four open reading frames (ORFs), one encoding for replication protein (rep) and the others encoding hypothetical proteins.

The resistome analysis identified the gene $b l a_{\text {POM-1 }}(98.78 \%$ id - accession number GU002295). No other antimicrobial resistance gene was found. One hundred and ninety-two (192) virulence factors were identified by VFDB. Genes encoding for quorum sensing system, antiphagocytosis, iron uptake, efflux pump, endotoxin and toxin, adherence, and secretion systems were detected (Table 1). From the 192 genes carried by strain $P$. otitidis K_25, 84.3\% are also present in $P$. aeruginosa. 
Table 1. Genomic and epidemiological features of the first meropenennonsusceptible Pseudomonas otitidis from chicken carcass at retail market in Brazil.

\begin{tabular}{cc}
\hline \multicolumn{1}{c}{ Features } & Pseudomonas otitidis $\mathrm{K}_{-} 25$ \\
\hline Genome data & \\
\hline Contigs $(>200 \mathrm{bp})$ & 6,4 \\
GC contente $(\%)$ & 735 \\
Number of genes & 67,06 \\
CDSs & 6.449 \\
tRNAs & 6.379 \\
\hline
\end{tabular}

Epidemiological Genomic Data

\begin{tabular}{|c|c|}
\hline Virulence factors ${ }^{\mathrm{a}}$ Class & Number of related genes \\
\hline Adherecence & 95 \\
\hline Antiphagocytosis & 29 \\
\hline Enzyme & 2 \\
\hline Iron uptake & 16 \\
\hline Protease & 1 \\
\hline Quorum sensing & 1 \\
\hline Regulation & 6 \\
\hline Secretion system & 25 \\
\hline Toxin & 2 \\
\hline Amino acid and purine metabolism & 1 \\
\hline Efflux pump & 1 \\
\hline Endotoxin & 1 \\
\hline Glycosylation system & 1 \\
\hline Immune evasion & 4 \\
\hline Invasion & 3 \\
\hline Iron acquision & 1 \\
\hline Magnesium uptake & 1 \\
\hline Serum resistance & 1 \\
\hline Stress adaptation & 1 \\
\hline Total & 192 \\
\hline \multicolumn{2}{|l|}{ Resistome $^{\mathrm{b}, \mathrm{c}}$} \\
\hline Beta-lactam ${ }^{\mathrm{b}}$ & $b l a_{\mathrm{POM}-1}$ \\
\hline
\end{tabular}

ahttp://www.mgc.ac.cn/VFs/; ${ }^{\text {bhttps://cge.cbs.dtu.dk/services/ResFinder/; }}$ chttps://card.mcmaster.ca/analyze/rgi

\section{DISCUSSION}

The genomic analysis revealed $b l a_{\mathrm{POM}-1}$ gene as the mechanism of meropenem non-susceptibility harboured by the $P$. otitidis strain (K_25) isolated from chicken carcass. The resistance gene $b l a_{\mathrm{POM}-1}$ encodes for the production of a metallo-beta-lactamase $(\mathrm{M} \beta \mathrm{L})$, which is known to confer resistance to carbapenems [4]. The gene $b l a_{\mathrm{POM}}$ seems to be highly conserved and constitutively expressed in P. otitidis [25]. While the expression of POM MBL does not necessarily confers a carbapenem-resistant phenotype in some $P$. otitidis strains, the strain P. otitidis K_25 reported in this study showed a reduced susceptibility to meropenem. Other resistance genes were not identified, and the strain was phenotypically susceptible to all other tested antimicrobials. As previously reported in other POM MBL-expressing strains [12], K_25 was susceptible to cephalosporins. This beta-lactam resistance profile is unusual in the Pseudomonas genus [12]. Pseudomonas otitidis was the first pathogenic Pseudomonas species constitutively expressing MBL in the absence of inducible beta-lactamase genes [25]. It has been suggested that the gene $b l a_{\mathrm{POM}}$ was originally acquired by horizontal transfer mechanisms, followed by recombination into the chromosome downstream of the conserved phosphonate operon, after divergence of $P$. otitidis from the other species [25].

Carbapenems are not administered to food animals [27] and resistance to this antimicrobial group is still low in bacteria isolated from foodstuffs and environment [3]. Still, spoilage Pseudomonas species showing resistance to carbapenems have been reported in pork, chicken and dairy products [18,28]. In Pseudomonas, carbapenem resistance is usually attributable to self-induced physiological changes triggered by several factors. The exposure to other antimicrobials has been suggested as a possible driver for the emergence of carbapenem resistance [3]. The origin and relevance of these carbapenem-resistant spoilage bacteria are still unclear. Most probably they are environmental bacteria that contaminate foodstuffs during processing and can reach the consumer through retail market. In the great majority of the cases, heat treatment of foods can destroy these contaminants and the proper handling of foods during preparation can mitigate their transmission to humans. Despite these aspects, the detection of viable $P$. otitidis harboring several virulence factors in chicken carcass at retail 
market highlights the potential role of spoilage bacteria as opportunistic pathogens [6]. Interestingly, $P$. otitidis K_25 shared $84.3 \%$ of the virulence genes harbored by $P$. aeruginosa, which is the reference pathogenic species that is able to colonize, evade the immune system and cause lesions in the human host [20]. These findings, corroborate the potential of P. otitidis K_25 to cause disease in humans.

The findings of the present study shed light on the potential role of spoilage bacteria in foodstuffs as opportunistic pathogens and reservoirs of antimicrobial resistance genes. Antimicrobial resistance should be addressed in a One Health perspective, as resistant bacteria and resistance determinants circulate among environment, animals and humans. In any of these interconnected compartments, bacteria are exposed to different selective drivers that affect fitness and evolution of strains, and these events may later reflect in the other compartments [14]. Moreover, in-depth genomic information of organisms in foodstuff, such as those provided in this investigation, can support evolutionary and epidemiologic studies addressing beta-lactam resistance among Gram-negative species, which can ultimately contribute to mitigate antimicrobial resistance in the animal-food industry.

\section{CONCLUSION}

The in-depth genomic characterization of a bla $a_{\text {POM-1 }}$-mediated meropenen-nonsusceptible $P$. otitidis $\mathrm{K} \_25$ strain from a chicken carcass highlights that spoilage bacteria in foodstuff can serve as reservoirs of genes conferring resistance against critically important antimicrobial drugs in human medicine.

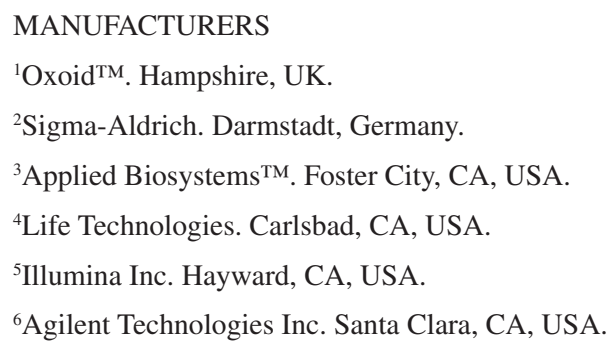

Declaration of interest. The authors report no conflicts of interest. The authors alone are responsible for the content and writing of this paper.

\section{REFERENCES}

1 Bankevich A., Nurk S., Antipov D., Gurevich A.A., Dvorkin M., Kulikov A.S., Lesin V.M., Nikolenko S.I., Pham S., Prjibelski A.D., Pyshkin A.V., Sirotkin A.V., Vyahhi N., Tesler G., Alekseyev M.A. \& Pevzner P.A. 2012. SPAdes: a new genome assembly algorithm and its applications to single-cell sequencing. Journal of Computational Biology. 19(5): 455-477.

2 Bao E., Jiang T. \& Girke T. 2014. AlignGraph: Algorithm for secondary de novo genome assembly guided by closely related references. Bioinformatics. 30(12): 319-328.

3 Bonardi S. \& Pitino R. 2019. Carbapenemase-producing bacteria in food-producing animals, wildlife and environment: A challenge for human health. Italian Journal of Food Safety. 8(2): 7956.

4 Borgianni L., De Lucca F., Thaller M.C., Chong Y., Rossolini G.M. \& Docquier J.D. 2015. Biochemical characterization of the POM-1 metallo- $\beta$-lactamase from Pseudomonas otitidis. Antimicrobial Agents and Chemotherapy. 59(3): 1755-1758.

5 Cabanettes F. \& Klopp C. 2018. D-GENIES: Dot plot large genomes in an interactive, efficient and simple way. PeerJ. 6: e4958. [Fonte: <https://peerj.com/articles/4958/>]

6 Clark L.L., Dajes J.J., McLean C.H., Bartell J.G. \& Stroman D.W. 2006. Pseudomonas otitidis sp. nov., isolated from patients with otic infections. International Journal of Systematic and Evolutionary Microbiology. 56(4): 709-714.

7 Clinical and Laboratory Standard Institute. 2018. Performance Standards for Antimicrobial Susceptibility Testing. 28th edn. CLSI supplement M100. Wayne, PA: Clinical and Laboratory Standard Institute.

8 Clinical and Laboratory Standard Institute. 2020. Performance Standards for Antimicrobial Susceptibility Testing. 30th edn. CLSI supplement M100. Wayne, PA: Clinical and Laboratory Standard Institute. 2020. Disponível em: < http://em100.edaptivedocs.net/GetDoc.aspx?doc=CLSI\%20M100\%20ED30:2020\&scope=user>.

9 Feng K., Li R., Chen Y., Zhao B. \& Yin T. 2015. Sequencing and analysis of the Pseudomonas fluorescens GcM5-1A genome: A pathogen living in the surface coat of Bursaphelenchus xylophilus. PLoS ONE. 10(10): e0141515.

10 Fernández M., Porcel M., De La Torre J., Molina-Henares M.A., Daddaoua A., Llamas M.A., Roca A., Carriel V., Garzón I., Ramos J.L., Alaminos M. \& Duque E. 2015. Analysis of the pathogenic potential of nosocomial Pseudomonas putida strains. Frontiers in microbiology. 6: 871. [Fonte: <https://www.frontiersin.org/articles/10.3389/ fmicb.2015.00871/full>]. 
11 Gurevich A., Saveliev V., Vyahhi N. \& Tesler G. 2013. QUAST: Quality assessment tool for genome assemblies. Bioinformatics. 29(8): 1072-1075.

12 Lee K., Kim C., Young D., Yum J.H., Chung M. \& Chong Y. 2011. POM-1 metallo- $\beta$-lactamase-producing Pseudomonas otitidis isolate from a patient with chronic otitis media. Diagnostic Microbiology and Infectious Disease. 72 : 295-296.

13 Liu B., Zheng D., Jin Q., Chen L. \& Yang J. 2019. VFDB 2019: A comparative pathogenomic platform with an interactive web interface. Nucleic Acids Research. 47(D1): D687-D692.

14 McEwen S.A. \& Collignon P.J. 2018. Antimicrobial resistance: a one health perspective. Microbiology Spectrum. 6(2): ARBA-0009-2017. DOI: 10.1128/microbiolspec.ARBA-0009-2017.

15 Nishiyama N., Uechi K., Nakamatsu M., Kinjo T. \& Fujita J. 2018. Three cases of POM-1 metallo- $\beta$-lactamase producing Pseudomonas otitidis isolated from respiratory specimens. Respirology. 23: 307-308.

16 Pang Z., Raudonis R., Glick B.R., Lin T.J. \& Cheng Z. 2019. Antibiotic resistance in Pseudomonas aeruginosa: mechanisms and alternative therapeutic strategies. Biotechnology Advances. 37(1): 177-192.

17 Quinn P.J., Markey B.K., Leonard F.C., Hartigan P.J., Fanning S. \& Fitzpatrick E.S. 2011. Enterobacteriaceae. In: Veterinary Microbiology and Microbial Disease. 2nd edn. Ames: Wiley-blackwell, pp.263-286.

18 Quintieri L., Fanelli F. \& Capuyo L. 2019. Antibiotic resistant Pseudomonas spp. spoilers in fresh dairy products: An underestimated risk and the control strategies. Foods. 8(9): E372. [Fonte: <https://www.mdpi.com/2304-8158/8/9/372/ $\mathrm{htm}>$ ].

19 Richter M., Rosselló-Móra R., Oliver Glöckner F. \& Peplies J. 2016. JSpeciesWS: A web server for prokaryotic species circumscription based on pairwise genome comparison. Bioinformatics. 32(6): 929-931.

20 Rocha A.J., Barsottini M.R.O., Rocha R.R., Laurindo M.V., Leandro F., Moraes F.L.L. \& Rocha S.L. 2019. Pseudomonas aeruginosa: virulence factors and antibiotic resistance genes. Brazilian Archives of Biology and Technology. 62: e19180503. DOI: 10.1590/1678-4324-2019180503.

21 Simpson J.T., Wong K., Jackman S.D., Schein J.E., Jones S.J. \& Birol I. 2009. ABySS: A parallel assembler for short read sequence data. Genome Research. 19(6): 1117-1123.

22 Stellato G., Utter D.R., Voorhis A., De Angelis M., Eren A.M. \& Ercolini D. 2017. A few Pseudomonas oligotypes dominate in the meat and dairy processing environment. Frontiers in Microbiology. 8: 264. DOI: 10.3389/ fmicb.2017.00264. [Fonte: <https://www.frontiersin.org/articles/10.3389/fmicb.2017.00264/full>].

23 Tan H., Zhang Z., Hu Y., Wu L., Liao F., He J., Luo B., He Y., Zuo Z., Ren Z., Peng G. \& Deng J. 2015. Isolation and characterization of Pseudomonas otitidis TH-N1 capable of degrading zearalenone. Food Control. 47: 285-290.

24 Taybali A.F., Coleman G. \& Nguyen K.C. 2015. Virulence attributes and host response assays for determining pathogenic potential of Pseudomonas strains used in biotechnology. PLoS ONE. 10(11): e0143604.

25 Thaller M.C., Borgianni L., Di Lallo G., Chong Y., Lee K., Dajes J., Stroman D. \& Rossolini G.M. 2011. MetalloLactamase Production by Pseudomonas otitidis: a Species-Related Trait. Antimicrobial Agents and Chemotherapy. 55(1): 118-123.

26 World Health Organization. 2018. Antimicrobial resistance (WHO Fact sheet). Geneva: World Health Organization; February. Disponível em: <http:// www.who.int/en/news-room/factsheets/detail/antimicrobial-resistance>

27 World Health Organization. 2019. OIE list of antimicrobial agents of veterinary importance. Geneva: World Health Organization. Disponível em: <https://www.oie.int/fileadmin/Home/eng/Our_scientific_expertise/docs/pdf/AMR/A_ OIE_List_antimicrobials_July2019.pdf>.

28 Wong M.H., Chan E.W. \& Chen S. 2015. Isolation of carbapenem-resistant Pseudomonas spp. from food. Journal of Global Antimicrobial Resistance. 3(2): 109-114.

29 Wu J., Jung B.G., Kim K.S., Lee Y.C. \& Sung N.C. 2009. Isolation and characterization of Pseudomonas otitidis WL-13 and its capacity to decolorize triphenylmethane dyes. Journal of Environmental Sciences. 21(7): 960-964. 